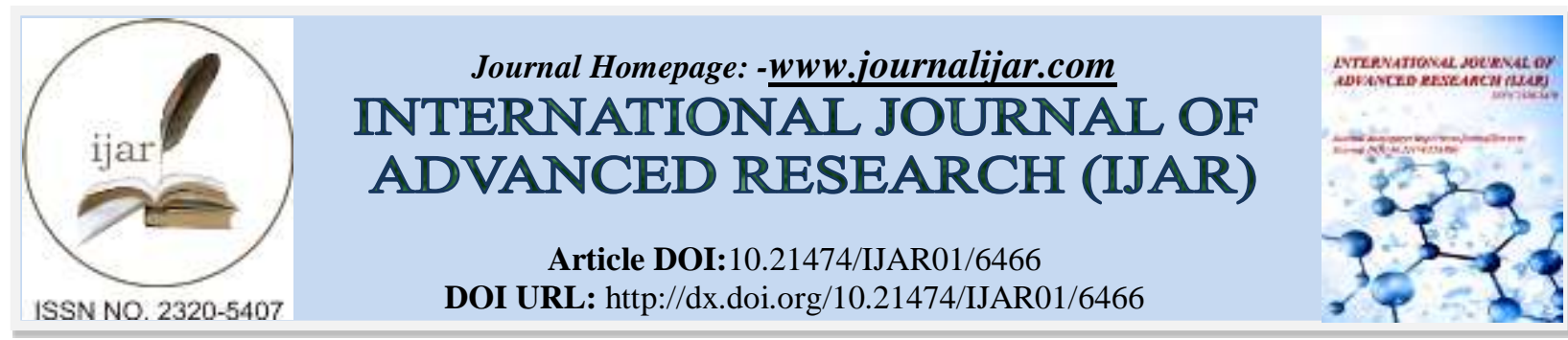

RESEARCH ARTICLE

\title{
AUTOIMMUNE HEPATITIS AS A RARE CAUSE OF HEPATITIS IN A HUMAN IMMUNE DEFICIENCY VIRUS INFECTED PATIENT ON ANTIRETROVIRAL TREATMENT: A CASE REPORT.
}

Vivek Kumar, Ajay Pratap Singh and Seetaram Singh Kulraj.

Department Of Medicine, King George's Medical University, Lucknow, India.

\section{Manuscript Info}

Manuscript History

Received: 07 December 2017

Final Accepted: 09 January 2018

Published: February 2018

Keywords:-

Human immunodeficiency virus, autoimmune hepatitis, interface hepatitis.

\section{Abstract}

The emergence of hepatic injury in patients with human immunodeficiency virus infection during highly active therapy presents a diagnostic dilemma. It may represent treatment side effects or Autoimmune disorders, such as autoimmune hepatitis, emerging during immune restoration. We present the case of a 25-year-old Indian woman with human immunodeficiency virus infection who presented to our emergency department with icterus, abdominal pain and was found to have autoimmune hepatitis. In our patient (the one patient case we are reporting), a liver biopsy revealed interface hepatitis, necrosis with lymphocytes and plasma cell infiltrates and variable degrees of fibrosis.

Copy Right, IJAR, 2018,. All rights reserved.

\section{Introduction:-}

Impaired immunity in individuals with human immunodeficiency virus (HIV) infection affects the defense mechanisms against pathogens and alters the regulation of autoimmunity [1]. This may lead to the emergence of autoimmune disorders or modification of pre-existing conditions. Several conditions may remit, such as systemic lupus erythematous (SLE), while others, such as psoriasis, intensify. The development of liver disease during highly active anti-retroviral treatment (HAART) in patients with HIV infection without evidence of co-infection with hepatitis viruses poses a diagnostic dilemma. This may be due to treatment side effects or to the emergence of autoimmune disorders during immune restoration. Autoimmune hepatitis (AIH) is rare in patients with HIV infection. Additionally, hepatic involvement is unusual in other common autoimmune disorders. We present the case of a patient with AIH and SLE emerging de novo during HAART and review all previously reported cases of AIH in patients with HIV infection who are undergoing HAART.

\section{Case Report:-}

A 25-year-old Indian woman who had been diagnosed with HIV infection in 2016 acquired by heterosexual contact and on Antoretoviral treatment(tenofovir, Lamivudine and Efavirenze) since then with the initial CD4 count of 516 cell $/ \mathrm{mm}$. In December 2017 her CD4 had been 958 cells $/ \mathrm{mm}$ and viral load undetectable. There was no personal or family history of autoimmune disease. Since December 2018 she started to experience gradual right upper quadrant pain associated with intermittent night sweats. She noticed yellowish discolouration of urine followed by her eyes during this time. She was seen in the emergency with deep icterus and right upper quadrant abdominal pain and was hospitalized.

Her physical examination revealed that she was afebrile and icterus was present with no remarkable finding on general examination. Her abdominal examination demonstrated diffuse abdominal tenderness that was most 
prominent in the right upper quadrant. A ultrasonography was done which showed mild hepatomegaly. Her routine blood investigation revealed jaundice with total bilirubin of $37.80 \mathrm{mg} / \mathrm{dl}$ (direct bil.=23.77mg/dl) and liver enzymes were deranged with SGOT $451 \mathrm{IU} / \mathrm{ml}$, SGPT 242IU/ml and Alkaline phosphate $306 \mathrm{IU} / \mathrm{ml}$. Her viral markers for Hepatitis B,C,A and E were negative. Assay for CMV and EBV were also negative. On day 3 of her admission a autoimmune profile was sent which came out to be positive with Anti-nuclear antibody 3+ DFS at 1:320, Anti Smooth Muscle Antibody 3+ at 1:160 and Immunoglobulin G of 3604mg/dl (normal 800-1800). A liver biopsy was planned which demonstrated a portal lymphoplasmacytic infiltrate with multifocal zones of hepatocellular centrilobular necrosis consistent with Autoimmune hepatitis Fig 1.

Given her clinical picture, her positive laboratory test for ANA, anti-SMA, elevated immunoglobulin G levels and the histopathology of her liver biopsy, a diagnosis of AIH was made. Her calculated AIH score was 17 (> 15 is considered a definite diagnosis according to the International Autoimmune Hepatitis Group criteria).

The patient was initiated on high-dose steroid therapy (50 mg daily). By the next two days, her abdominal pain had improved, and she was discharged from the hospital on a tapering dose of steroids. On follow up her Liver profile was improving and still is on tapered dose of steroids.

\section{Discussion:-}

In review of the literature revealed 12 cases of autoimmune hepatitis in patients with HIV infection[2-8]. Three had co-infections with hepatitis $\mathrm{C}$ virus and were receiving interferon therapy [6-8] and six more had AIH before starting HAART and one pediatric patient's data were missing. Only three patients developed AIH after starting HAART, similar to our patient.

All patients who received HAART prior to AIH had a significant rise in CD4 count and undetectable HIV RNA before AIH was diagnosed. It has been encountered in women who had significant elevations in CD4 count, suggesting the emergence of AIH during immune restoration. It presented insidiously with non-specific manifestations. The diagnosis is usually based on AIH score, the absence of other conditions and characteristic histopathological findings. The pathogenesis of liver damage in AIH in patients with HIV may be mediated by autoreactive CD4 or CD8 T-cells, or by an antibody-dependent cell-mediated cytotoxic response directed against liver antigens[9]. Interestingly, initiation of HAART has also been implicated in de novo AIH, and has thought to "unmask" AIH during the immune reconstitution phase following initiation of HAART treatment, mediated by a loss of peripheral tolerance and autoreactivity [10] Moreover, beyond serving as a catalyst, drugs can have a direct cytotoxic effect and lead to drug-induced $\mathrm{AIH}$, which is a well described clinical entity, though typically implicating minocycline or nitrofurantoin. In fact, the patient who presented with acute liver failure may have demonstrated this pathophysiology, with efavirenz serving as the catalyst in this clinical scenario. This is the prevailing theory, however, other cases have purported improvement of liver biochemistry tests following initiation of HAART, suggesting that the virus itself may somehow be the causative agent in certain instances[11]. The majority of reported cases in the literature support the former hypothesis, as most cases did not show improvement with HAART and required treatment with corticosteroids. The patients presented in this case also support this mechanistic theory.

\section{Conclusion:-}

Although a rare presentation, de novo AIH should be considered in patients with HIV, particularly in those with well-controlled disease where no other clear etiologies can be identified. A liver biopsy, albeit not mandatory, is very useful for diagnosis, and typically shows findings consistent with AIH, but this is not always the case. A combination of serologic, biochemical, clinical, and histologic data is needed to arrive at the diagnosis. Prompt treatment should be initiated, particularly in cases of worsening liver injury and evolving liver failure. Monitoring for infectious complications should be undertaken, but the risk appears to be minimal in patients with wellcontrolled HIV. 


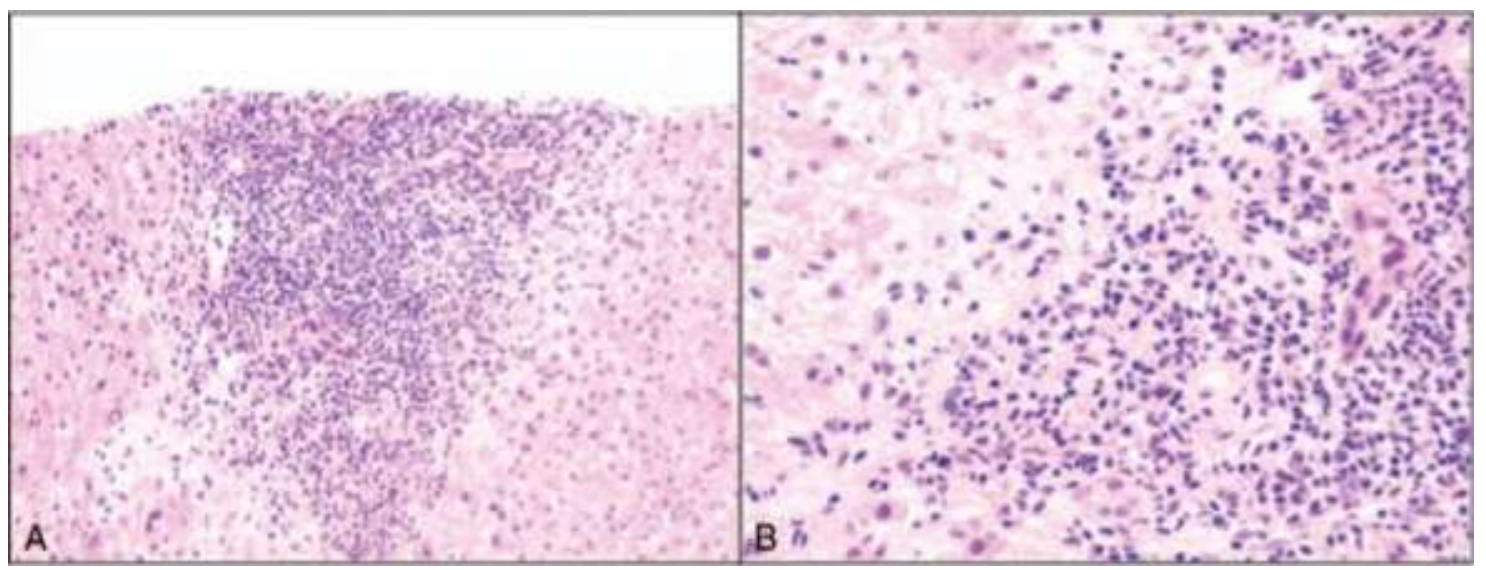

Fig 1:- A. low power microscope picture showing infiltrates in portal area. 1 B High power microscopic picture of dense plasma cell infiltrates.

\section{References:-}

1. Elhed A, Unutmaz D. Th17 cells and HIV infection. Curr Opin HIV AIDS. 2010;5:146-150. doi: 10.1097/COH.0b013e32833647a8.

2. German V, Vassiloyanakopoulos A, Sampaziotis D, Giannakos G. Autoimmune hepatitis in an

1. HIV infected patient that responded to antiretroviral therapy. Scand J Infect Dis. 2005;37:148

2. 151. doi: $10.1080 / 00365540510026841$.

3. Puius YA, Dove LM, Brust DG, Shah DP, Lefkowitch JH. Three cases of autoimmune hepatitis in HIV-infected patients. J Clin Gastroenterol. 2008;42:425-429. doi:

4. 10.1097/01.mcg.0000225591.08825.3e.

5. O'Leary JG, Zachary K, Misdraji J, Chung RT. De novo autoimmune hepatitis during immune reconstitution in an HIV-infected patient receiving highly active antiretroviral therapy. Clin Infect Dis. 2008;46:e12-e14.

6. Wan DW, Marks K, Yantiss RK, Talal AH. Autoimmune hepatitis in the HIV-infected patient: a therapeutic dilemma. AIDS Patient Care STDS. 2009;23:407-413. doi: 10.1089/apc.2008.0149.

7. Vispo E, Maida I, Moreno A, Barreiro P, Soriano V. Autoimmune hepatitis induced by pegylated interferon in an HIV-infected patient with chronic hepatitis C. J Antimicrob Chemother. 2008;62:1470-1472. doi: 10.1093/jac/dkn416.

8. Coriat R, Podevin P. Fulminant autoimmune hepatitis after successful interferon treatment in an HIV-HCV coinfected patient. Int J STD AIDS. 2008;19:208-210. doi: 10.1258/ijsa.2007.007185.

9. Cazanave C, Rakotondravelo S, Morlat P, Blanco P, Bonnet F, Beylot J. Autoimmune hepatitis in a HIV-HCV co-infected patient: diagnostic and therapeutic difficulties in French. Rev Med Interne. 2006;27:414-419. doi: 10.1016/j.revmed.2006.01.016

10. Diamantis I, Boumpas DT. Autoimmune hepatitis: evolving concepts. Autoimmun Rev 2004;3:207-14.

11. O'Leary JG, Zachary K, Misdraji J, et al. De novo autoimmune hepatitis during immune reconstitution in an HIV-infected patient receiving highly active antiretroviral therapy. Clin Infect Dis 2008;46:e12-14

12. German V, Vassiloyanakopoulos A, Sampaziotis D, et al. Autoimmune hepatitis in an HIV infected patient that responded to antiretroviral therapy. Scand J Infect Dis 2005;37:148-51. 